Vol. 7I, N. ${ }^{\circ}$ II3 (mayo 20I9), 75-90

\title{
RESEÑANDO ALGUNOS LÍOS DE LAS SUPUESTAS «CIENCIAS ECONÓMICAS»
}

\author{
JOHN CAJAS-GUIJARRO, ${ }^{1}$ KATHIA PINZÓN VENEGAS, ${ }^{2}$ BRYAN PÉREZ ALMEIDA ${ }^{3}$ \\ ${ }^{1}$ Universidad Central del Ecuador, ${ }^{2,3}$ Flacso, Ecuador
}

Recepción de manuscrito: 26 de abril de 2019

Aceptación de versión final: 30 de abril de 2019

\begin{abstract}
RESUMEn ¿Es la economía una ciencia? Probablemente no todavía. Con el objetivo de defender tal hipótesis, este artículo reseña algunos líos de las «ciencias económicas», como las diferencias entre una ortodoxia "científica» (pero, en realidad, reduccionista), y una heterodoxia «holista» (pero sin una síntesis que supere la negación de una ortodoxia indiferente), que mantienen a la economía dominada por mitos. Asimismo, se revisan algunos problemas de la noción ortodoxa de racionalidad: una concepción suprahumana del individuo que simplifica -en extremo- su representación matemática. Tales problemas dejan un agrio sabor a quien estudia «ciencias económicas» para realmente entender la realidad social.
\end{abstract}

palABRAS CLAVE Ciencias económicas, ortodoxia, heterodoxia, racionalidad, límites metodológicos.

ABSTRACT Is economics a «science»? Probably not yet. With the aim of defending such a hypothesis, this article reviews some «economic science» problems, such as the differences between a «scientific» orthodoxy (which is actually reductionist), and a «holistic» heterodoxy (but unable to propose a synthesis that goes beyond the contradiction against an apathetic orthodoxy), that keep economics dominated by myths. Also, some problems of the orthodox notion of rationality are reviewed: a supra-human conception of individuals used to - extremely- simplify their mathematical representation. These problems leave a bitter mouth-taste to those who studies «economic sciences» to truly understand social reality.

KEYWORDS Economic sciences, orthodoxy, heterodoxy, rationality, methodological limits.

JELCODES A11, B41, Y7o.

\section{INTRODUCCIÓN}

Alguna vez, la economista inglesa Joan Robinson dijo que:

Los economistas ortodoxos se han preocupado mucho por hacer exposiciones elegantes acerca de problemas de menor importancia, lo que distrae la atención de sus discípulos de las realidades desagradables del mundo moderno y el desarrollo de la argumentación abstracta ha ido más allá de la posible comprobación empírica. (1842, pág. 22) 
Esta frase engloba una realidad triste, pero cierta: la economía, como rama del pensamiento humano, más aún en su versión ortodoxa, está llena de problemas que ponen en duda su relevancia social. Dichos problemas se reflejan, por ejemplo, en la incapacidad de llegar a consensos ( $v$. gr., ortodoxia vs. heterodoxia), la existencia de mitos defendidos hasta en términos ideológicos (como la «mano invisible»), el uso de conceptualizaciones más instrumentales que explicativas (como la racionalidad neoclásica) y, en general, la incapacidad de explicar el sinfín de realidades desagradables que rigen en el mundo.

Semejante problemática - que incluso podría encerrar fuertes luchas de intereses y de poder - lleva a pensar que ante la pregunta de si se puede considerar a la economía como ciencia, habría razones para creer que aún no. Con el objetivo de argumentar a favor de esta hipótesis, el presente artículo reseña algunos líos de las «ciencias económicas» en respuesta a la urgencia de una reflexión necesaria no solo para mejorar la disciplina económica, sino sobre todo, para enfocar a las y los economistas en la búsqueda de soluciones a los problemas reales del mundo.

Así, luego de esta breve introducción (sección 1), este artículo plantea algunos puntos que ponen en duda la cientificidad de la economía, resaltando las disputas entre ortodoxia y heterodoxia (sección 2). Luego, el artículo centra su atención en la crítica a la noción neoclásica de racionalidad - fundamento de la corriente ortodoxa contemporánea- (sección 3) y, finalmente, se plantea una conclusión y una motivación para futuros aportes (sección 4).

\section{ECONOMÍA: UNA «CIENCIA» MUY PARTICULAR}

Rosa Luxemburg dijo alguna vez que la «economía política» es una «ciencia muy particular» llena de problemas y controversias, en donde ni siquiera los eruditos tienen una noción clara del «real objeto de su erudición» (Luxemburg, 1925, pág. 128). Si bien los años han pasado, esta crítica parece vigente no solo para la «economía política», sino, de forma más amplia, para todas esas caóticas áreas del pensamiento humano conocidas como "ciencias económicas». Ejemplo de los problemas que atraviesan dichas «ciencias» (y hasta ponen en duda su condición científica) son las diferencias, a ratos irreconciliables, entre las diversas escuelas del pensamiento económico — neoclásicas, neokeynesianas, monetaristas, neoinstitucionalistas, neoschumpeterianas, postkeynesianas, austriacas, marxistas, etc.- .

Antes de cuestionar la cientificidad de la economía, es necesario brindar al menos una idea de qué se puede entender por ciencia. En un sentido amplio, puede verse a la ciencia como un conjunto de teorías verificables, basadas en observaciones y evidencias que las respaldan, y un conjunto de prácticas y protocolos sistemáticos — recogidos en el método científico y sus etapas de observación, medida, experimentación, análisis de la información, replicación, revisión de pares, entre otras etapas- para generar medidas, categorizar la información, formular nuevas teorías y realizar experimentos. El objetivo de la ciencia y estos múltiples conjuntos que la conforman es obtener un conocimiento lógico, acumulable, racional y verificable, que permita reproducir los principales patrones de la realidad en el pensamiento, los cuales quedan recogidos en leyes científicas que suelen buscar en especial la predictibilidad de dichos patrones (poder predictivo) (Dahis, 2018, pág. 9; Bunge, 1997, págs. 6, 11-23). 
Aquella parte de la realidad sobre la cual la ciencia busca obtener un conjunto de conocimientos se suele llamar el objeto de estudio. Si ese objeto es una construcción mental ideal (como la lógica formal o la matemática pura) se puede hablar de una ciencia formal. En cambio, si el objeto de estudio compete a una parte de la realidad concreta (como la naturaleza o el ser humano) que, para su comprensión, requiere tanto de procedimientos empíricos (como la medición y cuantificación) como de construcciones conceptuales, entonces se tiene una ciencia factual (Bunge, 1997, págs. 8-9). En las ciencias formales, basta con que exista coherencia lógica para demostrar la veracidad del conocimiento adquirido; en cambio, en las ciencias factuales, la racionalidad y la lógica son necesarias, pero no suficientes para demostrar la veracidad del conocimiento adquirido; también se requiere de la verificación de ese conocimiento por medio de la observación y/o experimentación (ver Bunge, 1997, págs. 11-23).

Dentro de las ciencias, tanto formales como factuales, la divergencia entre escuelas del pensamiento no necesariamente es una debilidad; un ejemplo es el debate contemporáneo en física entre la teoría de cuerdas y la teoría de gravedad cuántica de bucles al tratar de explicar el vínculo entre el mundo micro (en donde rige la mecánica cuántica) y el mundo macro (en donde rige la relatividad). Sin embargo, mientras que en la física estas discusiones han sido antecedidas por un gran número de consensos, en la economía las divergencias siguen estancadas en los fundamentos. ${ }^{2}$ Así, la situación en economía se asemeja a un mundo paralelo donde coexisten múltiples interpretaciones de la mecánica de Newton negándose entre sí y siendo incapaces de llegar a consensos mínimos que permitan generar desarrollos más avanzados.

Quizá el resultado más notable de esas pugnas en las bases de las teorías es la existencia de dos grandes «corrientes» del pensamiento económico. Por un lado, existe una corriente principal ortodoxa (mainstream) compuesta de múltiples teorizaciones principalmente neoclásicas y neokeynesianas que suelen agruparse en los llamados modelos de equilibrio general dinámicos estocásticos (DSGEM por sus siglas en inglés). ${ }^{3}$ Por otro lado, emerge un sinfín de corrientes alternativas llamadas heterodoxas que suelen englobar (en un sentido erróneamente amplio) a todas las visiones que buscan construir sus propias teorizaciones con fundamentos distintos a los ortodoxos (Dequech, 2007); ${ }^{4}$ aquí han ganado notoriedad las corrientes poskeynesianas (ver Lavoie, 2014; Arestis, 1996), con modelos alternativos incluso a los DSGEM, como los modelos de consistencia flujo-stock (SFCM por sus siglas en inglés) (Godley y Lavoie, 2007). Tales son las pugnas entre las corrientes ortodoxas y heterodoxas que muchas veces ni siquiera debaten entre sí - de hecho, muy rara vez se retroalimentan y casi nunca llegan a consensossino que simplemente las unas niegan por completo la validez o relevancia de las otras, planteando diferencias incluso ontológicas (Lawson, 2005).

La lucha entre estas corrientes del pensamiento se visualiza en las críticas que cada una hace a la otra. A manera de ilustración, desde el lado heterodoxo, específicamente poskeynesiano, se puede tomar el ejemplo de Lavoie (2014), quien plantea cinco críticas a la ortodoxia, a saber: (1) La ortodoxia sería instrumentalista, pues no tiene interés en basar sus teorías desde representaciones explicativas de la realidad mientras las teorías hagan predicciones adecuadas, como sugeriría Friedman (1953) (ver más abajo). En oposición, la heterodoxia usaría una modelización (supuestamente) más empírica, realista y positiva antes que normativa (Berg y Gigerenzer, 2010). (2) La ortodoxia usualmente asumiría que el ser humano tiene información 
perfecta (y que existe conocimiento común), ${ }^{5}$ pudiendo llegar al extremo de conocer el propio modelo que supuestamente rige a la economía siguiendo una racionalidad modelo-consistente. ${ }^{6}$ En cambio, la heterodoxia asumiría que los individuos, en especial ante la incertidumbre, ${ }^{7}$ adoptan reglas simples de decisión basadas en la experiencia y sin gastar mucho tiempo ni recursos en ello - es decir, se acepta una aplicación heurística de reglas supuestamente reconociendo las limitaciones de la racionalidad humana al procesar información-. (3) Los modelos ortodoxos asumirían individuos optimizadores aislados, problema que crearía múltiples paradojas (v. gr., la «paradoja del ahorro») (Keynes, 1936; Dequech, 2007). En cambio, la heterodoxia adoptaría un enfoque más holístico reflejado, por ejemplo, en su mayor énfasis a temas como las instituciones ${ }^{8}$ y el poder ${ }^{9}$-incluyendo tanto análisis macro como microeconómicos-. ${ }^{10}(4)$ La ortodoxia asumiría que los recursos son escasos, de ahí su fijación en el "pleno empleo». Al contrario, la heterodoxia vería a los recursos como abundantes y se concentraría en su dinámica. (5) Para la ortodoxia los mercados serían los mejores mecanismos de asignación de recursos. En cambio, la heterodoxia cuestionaría la capacidad de autorregulación de los mercados y los vería con tendencias a la desestabilización - lo cual requeriría replantear la idea del «equilibrio» así como del papel del factor «tiempo»— ${ }^{11}$ (Dequech, 2007) ${ }^{12}$.

Con respecto al último punto, para Lavoie (2014, p.26), «la línea ortodoxa tiene una predilección conservadora, y debido a ello desea mostrar que las economías de mercado sin fricciones ni imperfecciones generan resultados eficientes, incluso si ello requiere supuestos absurdos».

En respuesta a las críticas que la heterodoxia ha hecho a la ortodoxia, esta última ha respondido simplemente con la indiferencia. Como plantea Colander (2010, pág. 47): «Mi respuesta honesta a la pregunta [ ¿¿Qué piensan los economistas del mainstream de la economía heterodoxa?», nota del autor] fue que ellos no piensan en ésta». De hecho, como señalan Gräbner y Strunk (2018, pág. 8), entre 1989 y 2008 menos del 3\% de las citas hechas en las trece revistas ortodoxas de mayor impacto en economía mencionan a las trece revistas heterodoxas más influyentes; en contraste, en esas trece revistas heterodoxas casi el 50\% de las citas recogen a las trece revistas ortodoxas más fuertes. Asimismo, como señala Lee (2009, citado en Carranza, 2018, pág. 222), entre 1993 y 2003 en la revista American Economic Review existieron un total de 36.336 citas de las cuales solo 23 correspondieron a revistas heterodoxas ${ }^{13}$. En otras palabras, los ortodoxos casi ni citan a los heterodoxos ${ }^{14}$, mientras que estos últimos sí suelen citar a sus «rivales». En este sentido, Lee (2012) sugiere que hay críticas ortodoxas señalando que los economistas heterodoxos deberían considerar su reputación académica y tratar de ingresar con sus ideas en el esquema ortodoxo-neoclásico, pues lo opuesto muy probablemente no sucederá. De hecho, el propio Lavoie (2014) plantea que quizá la heterodoxia no será una corriente principal (al menos por ahora), pues carece de una teorización única, observación con la que coincide Dequech (2007). Estey (1936), ya hace algún tiempo, mencionó además que la heterodoxia es difícil de definirla en términos no negativos - es decir, como lo que no es: ortodoxia-.

Semejante tipo de «discusiones» que más parecen «monólogos» —-donde la heterodoxia critica y la ortodoxia ni se inmuta-, dan cuenta de la limitada condición científica de la economía, si es que se puede hablar de dicha condición. Por ejemplo, las diferentes interpretaciones dadas a la «teoría general» de Keynes (1936) y sus derivaciones —en especial sobre la dinámica del desempleo, la causalidad entre ahorro e inversión, el rol de la «demanda efectiva» 
en el corto y el largo plazo o la disputa entre las teorías del dinero exógeno y el dinero endógeno- ${ }^{15}$ a criterio de Fontana y Gerrard (2006, pág. 72) muestran que la economía vive una etapa inmadura en donde «temas fundamentales todavía se encuentran en disputa».

Aparte de estas inmadureces, las «ciencias económicas» poseen otro problema quizá hasta más grave: los mitos y las analogías simplonas suelen dominar tanto en bandos ortodoxos como heterodoxos - y más aún entre economistas "prácticos» que reniegan de las teorías-. ${ }^{16}$ Un ejemplo típico es el mito de que existe una «mano invisible» que regula al mercado y lo lleva a una situación óptima cuando se la deja actuar libremente. Varios miembros del mainstream suelen defender ese mito citando a Adam Smith - pese a que él solo usó el término por tres ocasiones en todas sus obras, y una sola vez en la Riqueza de las Naciones (Samuels y cols., 2011, pág. 30-35) - y olvidando los casos concretos de concentración y centralización que suelen acompañar a la liberalización económica. ${ }^{17}$ Incluso, existe cierta evidencia de que las políticas de liberalización económica no siempre generan bienestar, sino que pueden complicar problemas como el crimen y la informalidad debido al potencial deterioro que podrían generar sobre el mercado laboral (Dix-Carneiro y Kovak, 2017; Dix-Carneiro y cols., 2018).

Otro mito dentro de las «ciencias económicas» es la idea de que los conceptos con los que estas trabajan están plenamente definidos. Un caso peculiar es el uso convencional que se da al concepto de «capital» en múltiples corrientes económicas (incluso heterodoxas) haciendo caso omiso de las fuertes controversias teóricas asociadas a dicho concepto. Aquí resalta la llamada "controversia de los Cambridge» o «controversia del capital», que en su primera fase puso en tela de duda la validez de la «curva de demanda de capital» (Sraffa, 1960; Samuelson, 1966; Cohen y Harcourt, 2003), y en su segunda fase cuestiona la unicidad y estabilidad de los modelos de equilibrio general de inspiración walrasiana (Fratini, 2019, págs. 12-13).

Los casos hasta aquí mencionados son apenas una muestra de todos los problemas que enfrentan las «ciencias económicas», y que (reiteremos) vuelven muy difícil su aceptación como verdadera ciencia. ${ }^{18}$ Ahora, considerando la hegemonía del mainstream en el pensamiento económico $-\mathrm{y}$ su indiferencia hacia la heterodoxia-, a continuación se reseñan algunos líos de la noción de racionalidad, uno de los «pilares ortodoxos fundamentales».

\section{ALGUNOS LÍOS DE LA RACIONALIDAD ORTODOXA-NEOCLÁSICA}

Dentro de la corriente ortodoxa contemporánea, los paradigmas neoclásicos -cuyas raíces más antiguas pueden hallarse en las corrientes marginalistas impulsadas por Jevons (1871), Menger (1871), Walras (1874) y la síntesis de Marshall (1890) — son dominantes, aunque en los últimos tiempos suelen compartir espacio con otros paradigmas ( $v . g r$., neokeynesianos). Tales paradigmas neoclásicos poseen varios líos. En particular, aquí se va a resaltar algunos de los problemas que afectan a la noción ortodoxa-neoclásica de racionalidad.

La noción de racionalidad forma parte de los paradigmas neoclásicos de mayor importancia en las corrientes ortodoxas. Antes de profundizar en esta noción, cabe aclarar que para la ortodoxia no necesariamente la racionalidad tiene un carácter ontológico, sino que, más bien, suele verse como una herramienta epistemológica y metodológica (en el sentido planteado por Dutt, 2015). Es decir, varios pensadores ortodoxos no asumen que efectivamente 
los individuos son racionales, sino que suponen la racionalidad como herramienta para tratar de replicar los patrones más generales del comportamiento individual. Por ejemplo, como señala Friedman (1953), para predecir la mejor forma de jugar billar se podría elaborar complejas fórmulas matemáticas - que tomen en cuenta factores físicos y demás- y luego usar esas fórmulas como aproximación para replicar cómo los jugadores de billar realizan sus tiros en la realidad; es decir, se asume que los jugadores actúan como si conocieran las fórmulas, aun cuando en realidad se tiene claro que no las conocen (teoría "como si»). ${ }^{19}$ Así, la verdadera importancia del supuesto radica en su carácter predictivo más que explicativo — aunque el carácter explicativo tampoco debería dejarse plenamente de lado en las intenciones neoclásicas a criterio de Sen (2008)-. ${ }^{20}$

Tal noción de racionalidad parte del supuesto de que los individuos tienen la capacidad de asignar un orden de preferencia ${ }^{21}$ a todas las opciones sobre las cuales se podría decidir; ese orden lo construyen usando relaciones de preferencia binarias (dos a dos) entre opciones, binaridad en palabras de Sen (2008, pág. 2). Asumida esa capacidad de comparación binaria, se considera que hay racionalidad cuando la relación de preferencia que construye el individuo cumple dos axiomas: completitud y transitividad. ${ }^{22}$

El axioma de completitud implica que, para todo par de opciones A y в, el individuo siempre puede definir que а es estrictamente preferida а в о viceversa о que а у в le resultan indiferentes (es decir, son igualmente preferidas). Así, la completitud implica que el individuo, ante cualquier par de opciones jamás cae en la indecisión, sino que siempre puede definir un orden de preferencia.

En cambio, el axioma de transitividad implica que, para toda tripleta de opciones A, B, C, si el individuo afirma que A es preferida а в у que в es preferida a c, entonces siempre deberá cumplirse para ese individuo que A es preferida a c. De este modo, la transitividad exige que el individuo, ante cualquier trío de opciones, jamás caiga en inconsistencias lógicas en sus órdenes de preferencia binarias. Es decir, si en un momento se dijo que a es preferida а в y luego se dice que в es preferida a C, entonces, por consistencia, nunca el individuo podrá afirmar que $\mathrm{C}$ es (estrictamente) preferida a A.

La noción de racionalidad es vital para las corrientes ortodoxas, pues se puede demostrar que solo cuando un individuo es racional (es decir, cumple con los axiomas de completitud y transitividad), entonces sus órdenes de preferencia pueden representarse en una función de utilidad (Mas-Colell y cols., 1995, pág. 9). Aquí la función de utilidad sirve para representar el orden de preferencias de un individuo usando números reales (es decir, la función cumple un rol ordinal). Por ejemplo, si un individuo prefiere А а в у в а с, una función de utilidad asignaría a la opción A un número real mayor (o igual) que el que asignaría a $\mathrm{B}$, y éste a su vez sería mayor (o igual) que el número asignado a $\mathrm{C}(e . g r ., \mathrm{U}(\mathrm{A})=1000, \mathrm{U}(\mathrm{B})=100, \mathrm{U}(\mathrm{C})=1$ donde no importa la magnitud de cada número siempre y cuando se cumpla con $\mathrm{U}(\mathrm{A}) \geq \mathrm{U}(\mathrm{B}) \geq \mathrm{U}(\mathrm{C})-{ }^{23}$

Así, la racionalidad es una noción clave que podría verse casi como la primera piedra de una pirámide invertida, desde la cual se edifica una parte relevante del paradigma neoclásico. Cabe decir esto, pues, con la racionalidad, dicho paradigma conecta la teoría de las preferencias individuales con la teoría de los números reales y el cálculo diferencial. ${ }^{24}$ Por cierto, semejante conexión no es nada sorprendente si se toma en cuenta que los axiomas de la racionalidad 
son en extremo análogos a los axiomas de orden de los números reales. En concreto, la completitud es análoga a la propiedad de tricotomía - para todo par de números reales $a, b$, siempre se cumple que $a>b$ o $b>a$ o $a=b$-, mientras que la transitividad es análoga a la propiedad transitiva - para toda tripleta de números reales $a, b$ y $c$, si $a>b$ y $b>c$, entonces siempre se cumple que $a>c$ - .

Esta analogía extrema entre los axiomas de racionalidad y los axiomas de orden de los números reales permite palpar una primera limitación: la noción neoclásica de racionalidad asume, de entrada, que las preferencias de los individuos son representables en el espacio de los números reales por medio de ordenamientos binarios, sin siquiera plantearse la posibilidad de que la forma como los individuos construyen sus preferencias quizá sea mucho más compleja y requiera otro tipo de representación. ${ }^{25}$

La posibilidad de que la racionalidad neoclásica sea insuficiente para reflejar la complejidad de las preferencias individuales se evidencia en varios casos donde la completitud y la transitividad no se cumplen. Respecto a la completitud, como se dijo antes, su cumplimiento depende de la capacidad de los individuos de siempre poder asignar un orden de preferencia a dos opciones, sin caer jamás en la indecisión. Semejante visión sería cuestionada por Von Neumann y Morgenstern (1944), quienes plantearían la existencia de opciones incomparables a las cuales los individuos simplemente no pueden asignar un orden de preferencia - lo cual, a su vez, requeriría el uso de una representación matemática más compleja-. Aumann (1962) reforzaría este argumento indicando que las opciones son incomparables cuando el individuo simplemente no es capaz de plantear una decisión ante dos opciones (Al-Suwailem, 2019, págs. 5-6). ${ }^{26}$ En el caso de la transitividad, podrían también existir múltiples razones para su no cumplimiento. Un ejemplo es la inconsistencia en las preferencias que puede surgir cuando las opciones poseen diferencias casi imperceptibles o cuando la capacidad de decisión es muy sensible al marco de referencia (Kahneman y Tversky, 1984) desde el cual se presentan las opciones (Mas-Colell y cols., 1995, págs. 7-8).

Otro problema de la noción neoclásica de racionalidad está en el supuesto de que las preferencias son exógenas: el orden de preferencia binaria de los individuos está predefinido y se mantiene intacto ante cambios en el contexto económico. Así, cuando un individuo se enfrenta a dos opciones А у в у escoge A cuando bien pudo escoger в, entonces el individuo «revela» su preferencia y - por consistencia, es decir, por transitividad- mantendrá esa preferencia estable en el tiempo sin importar si cambian los precios, la distribución del ingreso, las dotaciones iniciales de riqueza u otra variable económica. De hecho, la única situación en la que el mencionado individuo elegiría B en vez de A se daría cuando A no es económicamente alcanzable ( $v . g r$., su precio sube y rebasa el presupuesto del individuo); en ese caso, A sigue siendo preferida а в, pero, por limitaciones presupuestarias, в es elegida. En otras palabras, las preferencias del individuo son tratadas como exógenas ante el contexto económico, a diferencia de las decisiones efectivas que se tratan como endógenas ante dicho contexto - $\mathrm{y}$ reflejan parte de esas preferencias- ${ }^{27}$

Por tanto, si en algún momento el individuo antes mencionado cambia su preferencia y pasa a elegir B en vez de A cuando ambas opciones son alcanzables, entonces el supuesto de racionalidad y las preferencias exógenas implican que ese cambio se debió a algún aspecto 
extraeconómico, $v$. gr., cambio en las costumbres, en los gustos, en la cultura, entre otros elementos que el paradigma neoclásico asume como dimensiones donde la economía y el mercado no tendrían ninguna influencia. Esto denota una clara visión reduccionista de cómo los individuos cambian sus preferencias, pues deja de lado cuestiones como la publicidad y demás mecanismos de mercado que explícitamente buscan alterar ( $\mathrm{y}$ volver endógenas) las preferencias individuales.

Estas limitaciones del supuesto neoclásico de preferencias exógenas han sido criticadas casi desde los inicios del paradigma neoclásico-marginalista. En especial, es oportuno resaltar la crítica planteada por Veblen (1898):

En todas las formulaciones recibidas de la teoría económica [...] el material humano del que se ocupa la investigación se concibe en términos hedonistas; es decir, en términos de una determinada naturaleza humana pasiva y sustancialmente inerte e inmutable. [...] La concepción hedonista del ser humano es la de un calculador fulgurante de placeres y de pensar, que oscila como un glóbulo homogéneo de deseo y de felicidad bajo el impulso de los estímulos que le rozan la superficie, pero que le dejan intacto. No tiene antecedente ni consecuente. Es un dato humano aislado, definitivo, en equilibrio estable, excepto por los golpes de las fuerzas que le desplazan en una u otra dirección. Autosuspendido en un espacio elemental, gira simétricamente en torno a su propio eje espiritual hasta que el paralelogramo de fuerzas se abate sobre él, momento en que sigue la línea resultante. Cuando se agota el impacto, vuelve al reposo, como un glóbulo de deseo autosuficiente, como antes. (págs. 389-39o)

Una limitación adicional que vale destacar es la forma cómo el paradigma neoclásico intenta extender su noción de racionalidad individual a una racionalidad grupal. En concreto, el paradigma neoclásico suele adoptar el supuesto del agente representativo: asume que todos los individuos tienen preferencias iguales a las de un único individuo promedio, lo cual permite representar al comportamiento agregado como la sumatoria de comportamientos individuales idénticos. El objetivo de dicho supuesto suele ser el de simplificar la modelización matemática, la cual suele volverse en extremo compleja cuando se intenta lidiar con individuos que poseen preferencias distintas.

Así, es común que el paradigma neoclásico asuma que todos los individuos son racionales y, por tanto, sus decisiones grupales también cumplan con los axiomas de completitud y transitividad. En este sentido, la construcción de una decisión grupal requiere un conocimiento absoluto de las preferencias de todos los individuos y, a su vez, depende de los incentivos económicos disponibles. Sin embargo, experimentos como los desarrollados por Fey y cols. (1996) y McKelvey y Palfrey (1992), indican que el altruismo y la reputación - ambos aspectos sociales que trascienden al individuo- son factores que también intervienen en el desarrollo de los agentes y en su construcción de preferencias. ${ }^{28}$ Otro lío grave que emerge al tratar de reducir la elección social a la mera suma de preferencias individuales, sería resaltado por Arrow (1950), quien demostraría que es imposible agregar preferencias individuales heterogéneas de forma «satisfactoria ${ }^{29}$ a menos que se acepte la existencia de un «dictador» - es decir, de un agente capaz de provocar que sus preferencias individuales sean exactamente iguales a las preferencias decididas por el grupo-. 
Todas las críticas mencionadas sobre la racionalidad neoclásica — que son apenas una breve muestra de múltiples problemas que se dejan para una reflexión futura, como el problema de la enorme generalidad y hasta incompletitud del equilibrio general,,$^{30}$ la falta de representación de los procesos de negociación que llevan al equilibrio ${ }^{31}$ o la propia «controversia del capital»—, muestran que la ortodoxia económica, en definitiva, tiene pies de barro y camina en medio de un lodazal.

\section{CONCLUSIÓN}

Las divergencias existentes en las «ciencias económicas» - fundamentalmente entre corrientes ortodoxa y heterodoxa - se han estancado en las bases mismas de la teorización económica, lo cual evidencia una «inmadurez» de esta disciplina que ni los economistas más «eruditos» son capaces de resolver satisfactoriamente. Todo esto se ha enmarcado en un entorno donde no se entablan diálogos entre corrientes económicas y (consecuentemente) mucho menos, se llega a consensos.

El panorama actual reflejaría la pugna, por una parte, de una ortodoxia — autoproclamada a la fuerza y reforzada permanentemente como dominante - instrumentalista, que concibe a los individuos como poseedores de información mayormente perfecta y completa, de concepción «atomística», que tiene a la noción de escasez como regla general, y que mantiene su fe en la existencia de mercados con capacidad de autorregulación. Por otra parte, en la pugna emerge una heterodoxia que se autoproclama más realista, apegada a la existencia de incertidumbre fundamental, holística, menos dependiente de la noción de escasez y con una concepción «más compleja» - pero muchas veces menos formalizada- de los mercados. Mientras cada bando se proclama como poseedor de la razón casi sin escuchar al otro - con una ortodoxia conservadora cuya respuesta a la crítica heterodoxa es simplemente la indiferencia, mientras que la heterodoxia no logra consolidar una teoría única que rebase la mera negación de la ortodoxia-, los mitos y las analogías simplonas siguen dominando en el pensamiento económico (tanto teórico y aplicado), dejando hasta en el olvido debates medulares (como la controversia del capital).

Estos casos son apenas una muestra de todos los problemas que enfrentan las «ciencias económicas», y que ponen en duda su condición científica. Esa duda se refuerza aún más al revisar algunos líos que posee la noción de racionalidad, uno de los «pilares ortodoxos fundamentales», entendida en términos reduccionistas y binarios, anteponiendo la capacidad predictiva a la explicativa. Dicha noción pretende representar a las preferencias humanas en el limitado espacio de lo numérico, vínculo que exige suponer un ser humano que nunca cae en indecisiones, posee memoria-consistencia-conocimiento perfectos $y$, en definitiva, nace con una serie de capacidades suprahumanas. Para colmo, esa misma visión individual se busca trasladar a los grupos humanos, intento que se vuelve imposible a menos que se acepte el surgimiento de un individuo-dictador que decida por toda la sociedad.

Semejantes problemas teóricos, junto con muchos otros que dejamos para una reflexión futura - como el problema de la enorme generalidad del equilibrio general, la falta de representación de los procesos de negociación que llevan al equilibrio o la propia «controversia del 
capital»—, dejan un agrio sabor a quien desea estudiar «ciencias económicas» con el auténtico objetivo de entender la realidad social, ${ }^{32}$ y refuerzan la lección dejada por Joan Robinson: «El propósito de estudiar economía no es el de adquirir un conjunto de respuestas prefabricadas para los problemas económicos, sino aprender a no ser timado por los economistas».

\section{NOTAS}

1 Las «ciencias económicas», sobre todo ortodoxas, han logrado ubicarse (muy posiblemente para mal) en el nivel más alto de jerarquía en comparación a las demás «ciencias sociales» (Acosta, 2015; Fourcade et al., 2015; Ramos Silva, 2000), al punto de obtener un (discutible) premio Nobel (Acosta y Cajas-Guijarro, 2018). Tal ubicación la ha alcanzado, entre otras razones, por su énfasis en el uso del razonamiento formal matemático en su comprensión del comportamiento de los agentes económicos maximizadores y el uso intensivo de la estadística y la econometría para vincular sus argumentos teóricos con información empírica (Fourcade et al., 2015; Ramos Silva, 2000). Ese enfoque metodológico incluso ha logrado extenderse hacia otras «ciencias sociales», como las ciencias políticas, en donde la noción del agente racional maximizador se usa para entender, por ejemplo, procesos electorales u otras decisiones políticas (ver, por ejemplo; Swedberg, 2001; Whitely y Seud, 1996). Como ejemplo de esta jerarquía adquirida por economía se puede destacar que más del $40 \%$ de las citas en la revista American Economic Review (referente en la economía ortodoxa) provienen de las veinticuatro revistas restantes de mayor prestigio en economía, mientras que solo o,8\% y o,3\% de citas corresponden a las veinticinco revistas más prestigiosas de sociología y ciencias políticas respectivamente (Fourcade et al., 2015, citado en Carranza, 2018, pág. 228). 2 Un ejemplo está en las diferencias extremas entre las teorías del valor marxistas y marginalistas.

3 Por lo general, los modelos de equilibrio general dinámicos-estocásticos (DSGEM) incluyen los siguientes supuestos (que pueden considerarse como descriptivos de la economía ortodoxa contemporánea): agentes representativos optimizadores en términos intertemporales; comportamiento agregado «microfundamentado» en las decisiones de esos agentes; «equilibrio general» en el sentido del análisis simultáneo de mercados - los cuales incluso pueden tener fallas, $v$. gr., asimetrías de información-; se acepta la existencia de «rigideces» nominales en variables como salarios y demás precios; en el corto plazo, dichas rigideces crean brechas entre demanda y oferta agregada, generando inflación; asimismo, hay espacio para la política monetaria bajo la forma de un banco central que debe definir la tasa de interés nominal para estabilizar la inflación; además en el largo plazo el crecimiento depende de la acumulación de capital — siguiendo lógicas a la Solow-Swan-Ramsey-; el ahorro no iguala directamente a la inversión, sino que la inversión se decide según, por ejemplo, la rentabilidad o las necesidades futuras de «capital» (función de inversión); los mercados financieros aseguran que los individuos se apropien (y consumen intertemporalmente) los resultados de la inversión (Vines y Willis, 2018; Stiglitz, 2018).

4 La distinción ortodoxia-heterodoxia puede volverse muy compleja. Por ejemplo, según Lavoie (2014), es factible distinguir entre «disidentes ortodoxos» $\mathrm{y}$ «disidentes heterodoxos» dependiendo de su nivel de apego al mainstream. A su vez, se suele caer en la generalización de incluir en la misma categoría de «heterodoxia» a corrientes demasiado heterogéneas como poskeynesianos, marxistas, institucionalistas, economistas feministas, ecologistas, modelización basada en agentes y hasta corrientes de economía conductual. Paradójicamente, en el caso de la economía conductual -enfocada en sus inicios a encontrar, desde la psicología, patrones opuestos a la racionalidad-, la ortodoxia ha ido modificando «ligeramente» sus modelos de optimización para matematizar e incorporar dichos patrones psicológicos (Gabaix, 2014; Thaler, 2018).

5 Sobre el nivel de conocimiento que el paradigma neoclásico asume respecto a los individuos, también existen varias polémicas. Apenas por citar un ejemplo (dentro de la teoría de juegos) se encuentran las discusiones sobre el «juego del ciempiés» de Rosenthal (1981) y hasta qué punto los jugadores racionales llegan o no a jugar todos los turnos (Bornstein y cols., 2004). 
6 Un ejemplo extremo son las expectativas racionales (Lucas, 1972).

7 Por incertidumbre se puede entender, en términos muy genéricos, a una situación donde no se conoce la probabilidad con la que pueden ocurrir diferentes eventos, o incluso se desconocen dichos eventos (Keynes, 1936, 1937; Knight, 1921).

8 Aunque hay enfoques heterodoxos que intentan estudiar sobre todo la endogeneidad de las instituciones, Lavoie (2014) reconoce que todavía faltan aportes para una mejor explicación de éstas.

9 Cabe aclarar que la economía ortodoxa-neoclásica sí ha prestado también atención a la discusión sobre el poder. Un ejemplo es el trabajo de Bartlett (1989).

10 Para el caso particular de las corrientes poskeynesianas, hay algunas críticas por su potencial falta de microfundamentos para sus teorizaciones macro (Schoder, 2017).

11 Para algunas reflexiones heterodoxas sobre el rol del tiempo y la insuficiencia de la noción de equilibrio en economía, ver Robinson (1974, 1975).

12 Respecto a la teoría de la firma, los heterodoxos hacen una crítica a la corriente ortodoxa-neoclásica aduciendo que ésta deja de lado la influencia del tamaño ( $y$, consecuentemente, del poder) de la firma en su entorno económico, social y político, las implicaciones distributivas de la disyuntiva gestión/propiedad, y el rol de la concentración y estructura de mercado en la evolución de los costos marginales (Dequech, 2007). Para una visión alternativa de la firma, vista como una coalición política, ver March (1962). 13 Para una revisión crítica de la heterodoxia postkeynesiana desde una revista ortodoxa (de las pocas disponibles), ver el trabajo de Crotty (1980).

14 Entre los aspectos que podría explicar la poca atención dada a la heterodoxia por las revistas ortodoxas de mayor impacto podría estar el hecho de que las corrientes heterodoxas — usando a ratos formalizaciones matemáticas aún más complejas que las ortodoxas- no suelen usar las herramientas metodológicas convencionales de la ortodoxia neoclásica —como las técnicas de optimización estática e intertemporal- (Lavoie, 2014; Gräbner y Strunk, 2018).

15 Para los enfoques monetaristas, lo común es pensar que la masa monetaria es generada exógenamente y que, en el largo plazo, dicha masa es neutral en el sentido de que no tiene efectos sobre la economía real (Friedman, 1968). En oposición a esos enfoques existe la visión del dinero endógeno y su no neutralidad, bajo las cuales la masa monetaria se define endógenamente dependiendo de la actividad económica (particularmente el crédito) a la vez que la política monetaria puede tener efectos reales tanto en el corto como en el largo plazo (Lavoie, 2014, cap. 4). Aquí cabe destacar el énfasis dado por las corrientes poskeynesianas a la discusión monetaria (Harvey, 2016), junto con algunos elementos del enfoque del dinero endógeno que han sido adoptados (implícitamente) por las corrientes ortodoxas contemporáneas (v. gr., Romer, 200o). Para una breve discusión monetaria sobre endogeneidad y neutralidad del dinero, ver Cajas-Guijarro (2017).

16 Economistas que, para Keynes (1936), terminan como esclavos de la teoría de algún pensador muerto. 17 Para una breve reflexión sobre el mito del libre mercado, ver Cajas-Guijarro (2018, págs. 13-19).

18 Para algunas reflexiones preliminares que también ponen en duda a la economía como ciencia, ver Dahis (2018).

19 También en defensa de la simplificación teórica que realiza la economía ortodoxa, Callon y Latour (2011) mencionan que ésta tiene por objeto - a través de discusiones sobre el método- el describir, prever y calcular tan completamente «como sea posible» la movilización de recursos y de agentes, la producción, el intercambio. Así se deja de lado (bajo el nombre de externalidades) todas aquellas cosas que en la práctica no pueden tomarse como parte de ese cálculo, pues de incluirlos sucedería que los intercambios no se definirían, que los cálculos no se terminarían nunca y que, finalmente, no sería factible conocer quién posee y quién saca provecho en cada intercambio. En otras palabras, la simplificación teórica ortodoxa sería una suerte de herramienta que permite limitar como objeto de estudio de la economía a la «relación entre fines y medios escasos con usos alternativos» (Robbins, 1932, pág. 16). 20 Para una reflexión filosófica de hasta qué punto la racionalidad neoclásica puede o no interpretarse como una teoría explicativa, incluso de carácter psicológica, ver el trabajo de Satz y Ferejohn (1994). $21 \mathrm{Al}$ respecto, Sen (1973) menciona que incluso la adopción del término «preferencia» es erróneo pues 
implicaría un sentido estricto de primacía o superioridad, de una opción sobre otra para un individuo, lo cual, a su parecer, resulta demasiado ambicioso en consideración de que solamente las «decisiones» pueden llegar a ser visibles - y, por ende, susceptibles de modelización/teorización-.

22 Las primeras formulaciones cercanas a estos axiomas pueden encontrarse en el trabajo de Samuelson (1938). 23 La concepción de utilidad neoclásica tiene como raíz la noción «objetiva» de Bentham (1789, págs. 14-15) de que la utilidad es la «propiedad de todo objeto» de producir «beneficios, ventajas, placer, bien o alegría» o de evitar la «la malicia, el odio, el mal o la tristeza». Sin embargo, el paradigma neoclásico le da a esa noción una carga más «subjetiva», especialmente con Jevons (1871, págs. 43-44), para quien los objetos no tienen una utilidad en sí mismos, sino que ésta depende más de la percepción que tienen los individuos de cómo los objetos satisfacen sus necesidades. Actualmente, esta discusión del carácter subjetivo de la utilidad suele quedar olvidada bajo la teoría de las preferencias especialmente desde Samuelson (1938). Mishra (2008), por su parte, menciona que la noción de búsqueda de placer («utilidad») en sí misma carecería de sentido pues resulta ser, o imposible de comprobar, o demasiado general para ser refutada.

24 Sobre todo, si a los axiomas de completitud y transitividad se agrega el axioma de continuidad.

25 Ante esta limitación, existen intentos de representar matemáticamente el comportamiento individual usando enfoques diferentes al neoclásico -el cual se extiende incluso más allá del mercado, como la política-. Un ejemplo es el trabajo de Markey-Towler (2018) en donde, usando teoría de probabilidades, se intenta - aunque de forma no muy convincente - representar cuán probable es que una idea quede captada en la mente de una persona representando matemáticamente patrones psicológicos como la tendencia a la sugestión, la resistencia a ideas que van en contra de creencias centrales (Kelly, 1963) y la disonancia cognitiva (Festinger, 1957).

26 Para una crítica llamativa a la completitud, ver el ejemplo del asno de Buridán planteado por Sen (1973, pág. 248), en donde un asno debe decidir entre dos montones de heno para alimentarse, pero al no poder decidir cuál de los montones escoger pues ambos le gustan demasiado, finalmente el asno cae en ansiedad y muere de hambre - bajo un enfoque extremo de racionalidad, se diría que el asno prefirió morir de hambre a elegir uno de los montones de heno-.

27 Esa estabilidad de las preferencias es vital para sostener el axioma débil de las preferencias reveladas propuesto por Samuelson (1938) y que suele usarse para tratar de deducir la función de utilidad de los individuos desde sus elecciones efectivas. De hecho, se puede demostrar (bajo ciertas condiciones) la equivalencia entre el enfoque de las preferencias reveladas y la racionalidad (Samuelson, 1950), equivalencia que implícitamente requiere asumir la ecogenicidad de las preferencias ante cambios en los precios y el ingreso.

28 El propio dinero puede distorsionar (incluso psicológicamente) las preferencias de los individuos. Es más, al parecer el dinero tiene el potencial de cambiar la percepción de los individuos frente a la exclusión social, el dolor y hasta la muerte (Zhou y cols., 2009; Zaleskiewicz y cols., 2013).

29 Por satisfactoria, en concreto, se refiere al cumplimiento de dos condiciones: eficiencia de Pareto, es decir, si todos los individuos prefieren A a B, entonces en agregado el grupo también prefiere A a B; independencia a alternativas irrelevantes, es decir, si la preferencia de todos los individuos entre А у в no cambia aun cuando cambian sus preferencias por otras opciones, entonces la preferencia agregada del grupo entre А у в tampoco cambia - es decir, si los cambios en las preferencias por otras opciones son irrelevantes en términos individuales, entonces también lo son en términos grupales- (Arrow, 1950; Coleman, 1966).

30 Sobre el tema se recomienda ver Al-Suwailem (2019).

$31 \mathrm{Al}$ respecto, se puede ver el trabajo de Hodgson (1992).

32 Incluso se podría pensar que el estudio de las «ciencias económicas» genera una suerte de «alienación científica» en tanto que la supuesta "ciencia» cobra «vuelos propios» al reducir «a las personas a unos cuanto procesos unidimensionales o imponiéndoles servidumbres en nombre de una determinada idea de sistema "económico", "político", etcétera» (Naredo 2015, pág. 37, citado en Carranza, 2018, pág. 214). 


\section{REFERENCIAS}

Acosta, A. (2015). Las ciencias sociales en el laberinto de la economía. Polis, (41). Recuperado de https:// journals.openedition.org/polis/10917

Acosta, A. y Cajas-Guijarro, J. (2018). De las ciencias económicas a la post-economía. Reflexiones sobre el sin-rumbo de la economía. Ecuador Debate, (103), 37-59.

Al-Suwailem, S. (2019). The incompletability of markets. Recuperado de https://s3.amazonaws.com/academia.edu.documents/58170971/Market_Incompletability_12.01.2019.pdf?AWsAccessKeyId=AKIA IWOWYYGZ2Y53UL3A \&EXpires=1556050112\&signature=hW\%2BuIBTB\%2BOaTfXJfg9PDLKF88еA\%3D \&response-content-disposition=inline\%3в\%20filename\%3DThe_incompletability_of_Markets.pdf

Arestis, P. (1996). Post-Keynesian economics: towards coherence. Cambridge Journal of Economics, 2o(1), 111-135.

Arrow, K. (1950). A difficulty in the concept of social welfare. Journal of Political Economy, 58(4), 328-346.

Bartlett, R. (1989). Economics and power. Cambridge, uk: Cambridge University Press.

Berg, N. y Gigerenzer, G. (2010). As-if behavioral economics: Neoclassical economics in disguise? History of Economic Ideas, 18(1), 133-165.

Bornstein, G., Kugler, T. and Ziegelmeyer, A. (2004). Individual and group decisions in the centipede game: Are groups more «rational» players? Journal of Experimental Social Psychology, (40), 599-605.

Bunge, M. (1997). La ciencia. Su método y su filosofía. Recuperado de https://users.dcc.uchile.cl/ cgutierr/ cursos/INV/bunge_ciencia.pdf

Cajas-Guijarro, J. (2017). El suprapoder monetario. Rebelion.org. Recuperado de http://www.rebelion. org/noticia.php?id=235449

Cajas-Guijarro, J. (2018). Los capos del comercio. Concentración, poder y acuerdos comerciales en el Ecuador: un preludio. Quito, Ecuador: Plataforma por el Derecho a la Salud/Fundación Donum/Fos.

Callon, M. y Latour, B. (2011). «iNo calcularás!» o cómo simetrizar el don y el capital. Athenea Digital, 11(1), 171-192.

Carranza, C. (2018). Emergencias epistémicas de Economía Heterodoxa en Latinoamérica. (Tesis de doctorado). Recuperado de repositorio digital de la Flacso https://repositorio.flacsoandes.edu.ec/ bitstream/10469/14017/2/TFLACSO-2018CVCH.pdf

Cohen, A. J. and Harcourt, G. C. (2003, March). Retrospectives: Whatever happened to the Cambridge capital theory controversies? Journal of Economic Perspectives, 17(1), 199-214.

Colander, D. (2010). Moving beyond the rhetoric of pluralism: Suggestions for an 'inside-the mainstream' heterodoxy. En R. Garnett, E. Olsen and M. Starr (Eds.), Economic pluralism (pp. 36-47). London, UK: Routledge.

Coleman, J. S. (1966). The possibility of a social welfare function. American Economic Review, 56(5), 1105-1122.

Crotty, J. R. (1980). Post-keynesian economic theory: An overview and evaluation. American Economic Review, $70(2)$, 20-25.

Dahis, R. (2018). Is economics a science? well, not yet. Recuperado de https://papers.ssrn.com/sol3/papers.cfm?abstract id $=3036961$

Dequech, D. (2007). Neoclassical, mainstream, orthodox, and heterodox economics. Journal of Post Keynesian Economics, 30(2), 279-302. 
Dix-Carneiro, R. and Kovak, B. K. (2017, October). Trade liberalization and regional dynamics. American Economic Review, 107(10), 2908-2946.

Dix-Carneiro, R., Soares, R. and Ulyssea, G. (2018). Economic shocks and crime: Evidence from the Brazilian trade liberalization. American Economic Journal, 10(4), 158-195.

Dutt, A. (2015). Uncertainty, power, institutions, and crisis: implications for economic analysis and the future of capitalism. Review of Keynesian Economics, 3(1), 9-28.

Estey, J. A. (1936). Orthodox economic theory: A defense. Journal of Political Economy, 44(6), 791-802.

Festinger, L. (1957). A theory of cognitive dissonance. Stanford, usA: Stanford University Press.

Fey, M., McKelvey, R. D. and Palfrey, T. R. (1996). An experimental study of constant-sum centipede games. International Journal of Game Theory, 25(3), 269-287.

Fontana, G. and Gerrard, B. (2006). The future of post keynesian economics. Banca Nazionale del Lavoro BNL Quarterly Review, 59(236), (s. d.).

Fourcade, M., Ollion, E. and Algan, Y. (2015). The superiority of economists. Journal of Economic Perspectives, 29(1), 89-114.

Fratini, S. (2019). On the second stage of the cambridge capital controversy. Journal of Economic Surveys. doi:10.1111/joes.12312

Friedman, M. (1953). The methodology of positive economics. En M. Friedman (ed.), Essays in positive economics (pp. 3-43). Chicago, Usa: University of Chicago Press.

Friedman, M. (1968). The role of monetary policy. American Economic Review, 58(1), 1-17.

Gabaix, X. (2014, 09). A Sparsity-Based Model of Bounded Rationality. The Quarterly Journal of Economics, 129(4), 1661-1710.

Godley, W. and Lavoie, M. (2007). Monetary economics: An integrated approach to credit money, income, production and wealth. London, uk: Palgrave MacMillan.

Gräbner, C., y Strunk, B. (2018). Pluralism in economics: its critiques and their lessons. Documento de trabajo N. ${ }^{8}$ 2, Institute for Comprehensive Analysis of the Economy Johannes Kepler. Universidad de Linz. Recuperado de https://www.jku.at/fileadmin/gruppen/108/ICAE_Working_Papers/ wp82.pdf

Harvey, J. T. (2016). An introduction to post keynesian economics: Involuntary unemployment with perfectly flexible wages and prices. The American Economist, 61(2), 140-156.

Hodgson, G. M. (1992). The reconstruction of economics: Is there still a place for neoclassical theory? Journal of Economic Issues, 26(3), 749-767

Jevons, W. S. (1871). The theory of political economy. London, uk: Macmillan.

Kahneman, D. and Tversky, A. (1984). Choices, values and frames. American Psychologist, 39(4), 341-350.

Kelly, G. (1963). A theory of personality. Oxford, UsA: W. W. Norton.

Keynes, J. M. (1936). The general theory of employment, interest and money. London, uk: Palgrave Macmillan.

Knight, F. (1921). Risk, uncertainty, and profit. New York, USA: Dover Publications.

Lavoie, M. (2014). Post-keynesian economics: New foundations. Cheltenham, UK: Edward Elgar Publishing Limited.

Lawson, T. $(2005,12)$. The nature of heterodox economics. Cambridge Journal of Economics, 30(4), 483-505.

Lee, F (2009). A History of Heterodox Economics. Challenging the mainstream in the twentieth century. New York, usA: Routledge. 
Lee, F. (2012). Heterodox economics and its critics. Review of Political Economy, 24(2), 337-351.

Lucas, R. E. (1972). Expectations and the neutrality of money. Journal of Economic Theory, 4(2), 103-124. Luxemburg, R. (1925). The complete works of Rosa Luxemburg. Volume 1: Economic writings 1. London, UK: Verso.

March, J. G. (1962). The business firm as a political coalition. The Journal of Politics, 24(4), 662-678.

Markey-Towler, B. (2018). A formal psychological theory for evolutionary economics. Journal of Evolutionary Economics, 28(4), 691-725.

Marshall, A. (1890). Principles of economics. New York, usA: Cosimo Classics.

Mas-Colell, A., Whinston, M. and Green, J. (1995). Microeconomic theory. Oxford, usA: Oxford University Press.

McKelvey, R. D. and Palfrey, T. R. (1992). An experimental study of the centipede game. Econometrica, 6o(4), 803-836.

Menger, C. (1871). Principles of economics. Ludwig von Mises Institute.

Mishra, S. (2008, September). Structural changes in economics during the last fifty years. Documento de Trabajo n. ${ }^{\circ}$ 10534, University Library of Munich. Recuperado de https://ideas.repec.org/p/pra/ mprapa/10534.html

Naredo, J. M. (2015). La economía en evolución. Madrid, España: Siglo xxi editores.

Ramos Silva, J. (2000). Mathematics in economics: the competition point of view. Journal of Economic Studies, 27(4/5), 326-337.

Robbins, L. (1932). An essay on the nature and significance of Economic Science. London, UK: Macmillan.

Robinson, J. (1942). Ensayo sobre economía marxista. México DF, México: Siglo XxI.

Robinson, J. (1974). History versus equilibrium. En Collected economic papers (vol. 5, 48-58). Cambridge, UK: MIT Press.

Robinson, J. (1975). The unimportance of reswitching. The Quarterly Journal of Economics, 89(1), 32-39.

Romer, D. (2000). Keynesian macroeconomics without the LM curve. Journal of Economic Perspectives, 14(2), 149-169.

Rosenthal, R. W. (1981). Games of perfect information, predatory pricing and the chain-store paradox. Journal of the Operational Research Society, (25), 92-100.

Samuels, W., Johnson, M. and Perry, W. (2011). Erasing the invisible hand. Cambridge, Uk: Cambridge University Press.

Samuelson, P. (1938). A note on the pure theory of consumers' behavior. Economica, 5(1), 61-71.

Samuelson, P. (1950). The problem of integrability in utility theory. Economica, 17(68), 355-385.

Samuelson, P. (1966). A summing up. The Quarterly Journal of Economics, 8o(4), 568-583.

Satz, D. and Ferejohn, J. (1994). Rational choice and social theory. Journal of Philosophy, (32), 427-436.

Schoder, C. (2017). A critical review of the rationale approach to the microfoundations of post-keynesian theory. Review of Political Economy, 29(2), 171-189.

Sen, A. (1973). Behavior and the concept of preference. Economica, 40(159), 241-259.

Sen, A. (2008). Rational behavior. En The New Palgrave Dictionary of Economics (68-76). London, UK: MacMillan.

Sraffa, P. (1960). Production of commodities by means of commodities. Cambridge, UK: Cambridge University Press.

Stiglitz, J. (2018). Where modern macroeconomics went wrong. Oxford Review of Economic Policy, 34(12), $70-106$. 
Swedberg, R. (2001). Sociology and Game Theory: Contemporary and Historical Perspectives. Theory and Society, 30(3), 301-335.

Thaler, R. H. (2018, June). From cashews to nudges: The evolution of behavioral economics. American Economic Review, 108(6), 1265-87.

Veblen, T. (1898). Why is economics not an evolutionary science? The Quarterly Journal of Economics, 12(4), 373-397.

Vines, D. and Wills, S. (2018). The rebuilding macroeconomic theory project: an analytical assessment. Oxford Review of Economic Policy, 34(1-2), 1-42.

Von Neumann, J. and Morgenstern, O. (1944). Theory of games and economic behavior. Princeton, UsA: Princeton University Press.

Walras, L. (1874). Elements of pure economics. London, UK: Routledge.

Whitely, P. and Seud, P. (1996). Rationality and party activism: Encompassing tests of alternative models of political participation. European Journal of Political Research, (29), 215-234.

Zaleskiewicz, T., Gasiorowska, A., Kesebir, P., Luszczynska, A. and Pyszczynski, T. (2013). Money and the fear of death: The symbolic power of money as an existential anxiety buffer. Journal of Economic Psychology, (36), 55-67.

Zhou, X., Vohs, K. and Baumeister, R. (2009). The symbolic power of money: Reminders of money alters social distress and physical pain. Psychological Science, 20(6), 700-706. 